\title{
Operations research in transportation and supply chain management
}

\author{
M. Grazia Speranza
}

\begin{abstract}
The developments in digital technologies are creating new challenges and opportunities to Operations Research. In this paper, research trends in transportation and supply chain management will be discussed and some examples briefly presented.
\end{abstract}

Key words: Digital technologies, trends, research opportunities, transportation, supply chain management, logistics

\section{Introduction}

Information and communication technologies (ICT) are having a continuously increasing impact on our daily life and on all economic and social activities. With ICT we generally mean the infrastructure and components that enable modern computing. Although there is no single, universal definition of ICT, the term is generally accepted to mean all devices, networking components, applications and systems that combined allow people and organizations (i.e., businesses, nonprofit agencies, governments) to interact in the digital world.

Computers have been part of our daily life since the early eighties and the digitalization processes have proceeded at a regular pace over time since then. However, recently new phenomena, enabled by developments in digital technologies, are taking place and changing the economy and the society. Internet and all the technological devices that can receive and transmit data and information, including the mobile cellular phones, have enabled the design and introduction of new products and services, and new management styles. Companies with a long successful history have disappeared, replaced by new companies because businesses have completely changed. The behaviour of citizens, in the daily activities, keeps changing.

University of Brescia, Italy e-mail: grazia.speranza@unibs.it 
Expressions like Internet of things, big data, machine learning, sharing economy may not be clearly defined but indicate global phenomena. The fact that such expressions are regularly used in the media and by everyone is a sign of the high impact they are having. The Internet of things is the interconnection via the Internet of computing devices embedded in everyday objects, enabling them to send and receive data. Big data refers to data sets that are so big and complex that traditional dataprocessing application software are inadequate to deal with them. Machine learning is a subset of artificial intelligence in the field of computer science that often uses statistical and optimization techniques to give computers the ability to 'learn' (i.e., progressively improve performance on a specific task) with data. Sharing economy is an umbrella term with a range of meanings, often used to describe economic activities involving online transactions.

Transportation and logistics are two fields where Operations Research (OR) has substantially contributed in the last 50 years, especially improving the efficiency of operations but also providing support to decision making in strategic and tactical phases of the decision processes. The meaning of 'logistics' is slightly different from that of 'supply chain management'. Logistics refers to the planning, execution, and control of the procurement, movement, and stationing of personnel, material, and other resources to achieve the objectives of a plan, project, or strategy. Logistics may be defined as the management of inventory in motion and at rest. Supply chain management (SCM) is the broad range of activities required to plan, control and execute a product flow, from acquiring raw materials and production through distribution to the final customer. While logistics does not usually include the production phases that transform raw materials into a final product, supply chain management does not include the logistic operations in different areas such as the organization of events (for example, conferences or concerts). In this paper, we will refer to contributions to supply chain management but part of the discussion can be extended to logistics.

ICT is changing the processes in supply chain management and the way goods and people are transported, and this in turn is changing the problems where OR can contribute. May be it is also, at least partially, changing the domain of OR, pushing its boundaries.

\section{Supply chain management}

Supply chain management aims at integrating the various components of a supply chain and has been a research topic for OR for already some decades. Four major directions for research can be derived from the technological changes: a systemic, a collaborative, a dynamic and a data-driven direction. In the following we briefly overview the research opportunities associated with each of these directions. 


\subsection{Systemic direction}

Better solutions to problems can be identified when parts of a supply chain are jointly modeled and optimized. The digital technologies have enabled the implementation of integrated management styles. Research efforts in this direction have already been made recently. For example, in the area of vehicle routing, several papers have studied combined problems previously studied separately. Integrated vehicle routing problems (VRPs) is the expression increasingly used to denote the class of problems where routing decisions are taken jointly with other decisions (as outlined by the special issue edited by Bektaş et al. [9]). Location-routing problems jointly optimize location and routing. Inventory-routing problems combine routing and inventory management. Production-routing problems integrate production, routing, and inventory decisions. Multi-echelon routing problems optimize the routes of vehicles in distribution systems comprising two or more echelons. Routing problems with loading constraints simultaneously optimize the routing of vehicles and the loading of goods.

Integrated VRPs combine problems that are usually NP- hard when treated individually. However, solving the individual problems sequentially, even by means of exact methods, leads to a sub-optimal solution for the integrated problem, even if solved with a heuristic. The cost reduction achieved through the integration may be an order of magnitude greater than the cost reduction usually obtained by an exact method with respect to a heuristic. One of the first papers that showed the benefits of integrated decisions is due to Chandra and Fisher [11]. More recently, Archetti and Speranza [6] compared the heuristic solution of an inventory-routing problem with the solution obtained by sequentially and optimally solving the inventory management and the routing problems. The sequential solution models a traditional management style where customers of a supply chain control their inventory and decide order times and quantities. Then, the supplier organizes the distribution that, however, has to take order times and quantities as constraints. The inventory-routing problem models a more recent integrated policy, called Vendor Managed Inventory (VMI), where the supplier is responsible for the distribution and for the inventory at its customers. In [6] the results of computational tests show that solutions of the inventory-routing problem allow average savings of $10 \%$. The integration of production and inventory-routing is studied in [1]. The value of integrating loading and routing is analyzed in [13].

Integrated optimization problems model integrated management styles adopted in supply chain management, contribute to exploit the advantages of the integration and can quantify the benefits. The research opportunities in this direction are endless. 


\subsection{Collaborative direction}

Different forms of collaboration in supply chain management have been discussed, internal and external, horizontal and vertical. When dealing with road transportation, horizontal cooperation among carriers can be further classified according to the operational collaboration mode in order sharing and capacity sharing (see, for instance, the recent survey [20]).

Obviously, partners of a collaboration initiative aim at improving the performance of their own business. Each partner will be focused on its own business rather than on global performance. The collaboration initiative may bring benefits to some of the partners only or the benefits may be distributed in a way that is unacceptable to some of the partners. Thus, integration must be mediated with individual interests to make the collaboration initiative successful. This concept makes models for decision support in collaboration initiatives often different from models for global optimization.

Several statistics show that approximately $90 \%$ of freight travels on road. In European countries between $15 \%$ and $30 \%$ of trucks travel empty and contribute to traffic, pollution, accidents. The average load of a truck is much lower than its capacity, especially in city distribution. As a consequence, the number of trucks on road is much higher than it could be. Collaboration among carriers may improve the statistics and generate economic benefits for the carriers involved, a sector which lives on very low margins, as well as social and environmental benefits. A pilot European project, called 'Collaboration Concepts for Co-modality', has been implemented by two companies with production sites in Belgium and with large quantities of goods delivered to Greece. One of the two companies transports light large-volume goods, the other heavy low-volume goods. The project has achieved a reduction of 150,000 $\mathrm{km}$ travelled, and a reduction of $17 \%$ of the routing costs.

As an example, in Fernández et al. [14] a collaboration scheme is adopted by a consortium of carriers. Each carrier can decide which of its customers to serve and which ones to share with the other carriers. A shared customer can be served by any of the carriers. A carrier will tend to share customers not conveniently located and/or with low demand The revenue from a shared customer is partly collected by the 'owner' of the customer and partly by the one actually serving the customer. The optimization model proposed assigns the shared customers to carriers and builds the routes for all the carriers to serve all the customers in such a way that the total cost is minimized and each carriers has a profit at least as high as the profit it would have without collaboration. The computational results show that the profit increase in the collaborative setting strongly depends on the location and demand of customers, and ranges from small values to up to $85 \%$.

The research opportunities include the modelling and the solution of collaborative schemes. While imposing that the individual participants in a collaborative initiative gain with respect to a non collaborative scheme is a necessary condition for the individuals to join the initiative, it may not be a sufficient condition, as the profit may end up being distributed in an unfair way among the participants. 


\subsection{Dynamic direction}

The flow of data about customers, purchases, deliveries, locations, inventories, combined with the frequency of the update of the information, gives rise to a number of new dynamic problems. The phenomenon of e-commerce is in constant growth and is challenging all commercial companies and their logistics partners. The last-mile delivery problem remains a challenging problem to solve and logistic companies keep exploring new ways to offer a service to their customers that is up to their expectations. Additional transportation needs are set by the flow of on-line ordered goods that are returned, and in general by the reverse logistics.

Classical optimization models are based upon the assumption that information is available, that a model is solved and the solution implemented. This is a less and less realistic assumption. While until few years ago a delivery time of 5-10 days was acceptable to customers, today on-line customers wish and expect to receive the goods within the same day of the order or the day after. Moreover, they wish to choose the day, the time, the delivery point. The increased frequency and the reduction in quantities of the deliveries to shops and retailers make the urban logistics even more complex. The urban city logistics keeps changing because new and disruptive ways to tackle the problem are being experienced by the logistic companies, sometimes under constraints or incentives set of the public authorities.

Dynamic problems in transportation have been discussed for a long time (for example, by Psaraftis [18]), but research on dynamic and stochastic VRPs received increasing interest only in the last decade (see the recent survey by Ritzinger et al. [19] where the importance of appropriately modeling dynamic events and simultaneously incorporating information about the uncertainty of future events is outlined). Research opportunities related to the dynamism of supply chains are discussed in [21].

Another challenge to the OR community implied by the dynamism of processes is related to the computational times needed to find a solution to a problem. Also, long computational times may not be justified when the solution may be only partially implemented and the data is likely to change shortly after.

\subsection{Data-driven direction}

The quantity of data available is huge because of the devices that can collect and transmit data and because of the capacity of the systems that can store the data. Data creates enormous learning opportunities in all sectors. The amount of research in machine learning (see, for example, [3]) has been growing over the last years and various machine learning techniques have been successfully applied to a number of real life situations. Machine learning is related to mathematical optimization because several learning problems are modelled as optimization problems where the cost function, to be minimized, measures the discrepancy between the forecasts of the model and the real observations over time. A different relation with mathemat- 
ical optimization consists in using the machine learning techniques in optimization problems. Presentations on the use of machine learning techniques in transportation and supply chain management have started to appear in conferences but this line of research is still in its infancy.

\section{People transportation}

OR has contributed to the solution of many problems with big impact in people transportation, especially problems related to modes, air, railways, buses, for example, timetabling and crew scheduling. The way people move is, however, changing dramatically. Cars are becoming connected and, especially in the United States, huge research resources are invested on the development of autonomous vehicles. People expect to have flexible transportation services, with different levels of cost and comfort. Bike and car sharing are services already offered in most towns. In this field, we hear more and more the expression transportation as a service. Someone predicts that private cars will be owned in the future for the pleasure of driving and not for the need of people to be transported.

Public authorities need to study solutions that protect the well-being of citizens, challenged by high-frequency deliveries of small quantities of goods and cars with one passenger only. Space for the parking of cars and for the loading/unloading operations of vehicles is a scarce resource. The evolution towards electric vehicles will reduce pollution in urban areas but will not reduce the number of traveling vehicles, the need for space and the level of congestion.

From the OR perspective, the only optimization models OR contributed to private vehicles have been those aimed to optimize the shortest path from origin and destination, embedded nowadays in the several sat-nav systems available. The evolution of the way people are transported is opening new research directions to OR.

\subsection{On-demand services}

The number of traveling vehicles can be reduced only by reducing the number of people in need of travel and/or by increasing the number of people transported in the same vehicle. While OR can hardly contribute to the former option, contribution of OR may be relevant in supporting the latter.

The main reason that leads people to use their own vehicle is the lack of flexibility of mass transit systems. Such systems work on fixed itineraries and fixed schedules. Nowadays, for most potential customers the frequency is too low and the travel time is too high, with changes of transportation mean and waiting times. Moreover, those systems do not provide a transportation service from origin to destination. Taxis, on the other hand, are too expensive for regular use for most travelers. 
Demand Responsive Transit (DRT) systems (also called dial-a-ride systems) are flexible services that provide 'door-to-door' transportation. DRT systems have been mainly implemented as services for small groups of people (see [12] for a survey).

Martínez et al [17] have suggested a classification of DRT systems:

- with fixed itineraries and stops, with pre-booking;

- with fixed itineraries and stops with possible detours;

- with unspecified itineraries and predefined stops;

- with unspecified itineraries and unspecified stops.

An implementation of a DRT system in Maryland is presented by Marković et al. [16] and the benefits, with 450 trip requests daily, of a computerized routing and scheduling system are estimated with annual savings of $\$ 0.82$ million, or about $18 \%$ of the total annual expense, with respect to manual operations.

In [7] a simulation study is performed where a conventional mass transit system, say buses, is offered together with an on-demand service without fixed itineraries and schedules, provided through minibuses. A minibus, if acceptable to the user in terms of arrival time to destination, will pickup the user at the origin of the trip and deliver him/her to the destination. In case neither the conventional bus nor the ondemand minibus provide an acceptable service to the user, he/she will use a private car. A recent paper [8] introduced a Flexible Mobility On Demand (FMOD) system that offers different services, taxis, shared taxis and minibuses, where the minibus service works as a regular bus service with fixed schedules.

The so called dynamic ride-share systems share with the DRT systems the goal of increasing the number of people sharing the same vehicles. Such systems aim to bring together travelers with similar itineraries and time schedules on short-notice. Optimization methods that match drivers and riders in real-time are necessary for a successful implementation of such systems (see [2] for a review of dynamic ridesharing systems).

The need for parking space can be also reduced through car sharing systems, where a car is pre-booked, used and returned to a parking station. One-way, with respect to two-way, systems provide more flexibility to users since cars can be dropped-off at any station (see, for instance, [10]). Research opportunities include the location of stations and cars, car relocation problems, coordination of reservations.

\subsection{New shortest path problems}

As previously mentioned, sat-nav systems nowadays embed shortest path algorithms. Real-time information on traffic conditions imply the need of different algorithms that consider time-dependent uncertain travel times (see [15] for a recent survey).

Moreover, coordination opportunities among the routes of vehicles because of their capability of receiving and transmitting information. In [4] a model is proposed 
for the problem of coordinating the routes of vehicles in such a way that congestion is eliminated or reduced to a minimum level by keeping the inconvenience, measured as the increase of the traveling time with respect to the minimum possible, for cars below a given threshold.

\subsection{People and freight}

Collaboration opportunities are being explored by companies in all directions, including collaboration of people to transport goods with the goal of reducing the costs of a distribution process that is extremely expensive. The line between freight and passenger transportation, which was clearly defined until some time ago, is not any longer. An example is related to regular customers of shops who may become available, for a small compensation, to deliver goods to on-line customers on their way home. These customers are called occasional drivers (see, for example, [5]) and can contribute to make the delivery of goods to on-line customers more efficient.

\section{Conclusions}

New research opportunities keep arising because of new and disruptive options for transporting goods and people, enabled by the digital technologies. Lack of data has been an obstacle to the implementation of OR models and algorithms. Nowadays, data is huge in volume, variety, velocity and OR can exploit its potential, provided it will be capable of adapting to the new research challenges.

\section{References}

1. Absi, N., Archetti, C., Dauzère-Pérès, S., Feillet, D., Speranza, M.G. Comparing sequential and integrated approaches for the production routing problem, to appear in European Journal of Operational Research, DOI:10.1016/j.ejor.2018.01.052.

2. Agatz, N., Erera, A., Savelsbergh, M., Wang, X.: Optimization for dynamic ride-sharing: A review, European Journal of Operational Research 223 295-303, 2012.

3. Alpaydin E.: Introduction to machine learning, The MIT Press, 2014.

4. Angelelli, E., Arsik, I., Morandi, V., Savelsbergh, M., Speranza, M.G.: Proactive route guidance to avoid congestion, Transportation Research B 94 1-21, 2016.

5. Archetti, C., Savelsbergh, M., Speranza, M.G.: The vehicle routing problem with occasional rrivers, European Journal of Operational Research 254 472-480, 2016.

6. Archetti, C., Speranza, M.G.: The inventory routing problem: The value of integration, International Transactions on Operations Research 23 393-407, 2016.

7. Archetti, C., Speranza, M.G., Weyland, D.: On-demand public transportation, to appear in International Transactions on Operations Research, 2018. 
8. Atasoy, B., Ikeda, T., Song, X., Ben-Akiva, M.E.: The concept and impact analysis of a flexible mobility on demand system, Transportation Research Part C: Emerging Technologies 56 373-392 2015.

9. Bektaş, T., Laporte, G., Vigo, D.: Integrated vehicle routing problems, Computers \& Operations Research 55 126, 2015.

10. Bruglieri, M., Colorni, A., Lu, A.: The relocation problem for the one-way electric vehicle sharing, Networks 64 292-305, 2014.

11. Chandra, P., Fisher, M.L., Coordination of production and distribution planning, European Journal of Operational Research 72 503-517, 1994.

12. Cordeau, J.-F., Laporte, G.: The dial-a-ride problem: models and algorithms, Annals of Operations Research 153 29-46, 2007.

13. Côté, J.-F., Guastaroba, G., Speranza, M.G.: The value of integrating loading and routing, European Journal of Operational Research 257 89-105, 2017.

14. Fernández, E., Fontana, D., Speranza, M.G.: On the collaboration uncapacitated arc routing problem, Computers \& Operations Research 67 120-131, 2016.

15. Gendreau, M., Ghiani, G., Guerriero, E.: Time-dependent routing problems: A review, Computers \& Operations Research 64 189-197, 2015.

16. Marković, N., Nair, R., Schonfeld, P., Miller-Hooks, E., Mohebbi, M.: Optimizing dial-aride services in Maryland: Benefits of computerized routing and scheduling, Transportation Research Part C: Emerging Technologies 55 156-165, 2015.

17. Martínez, L.M., Viegas, J.M., Eiró, T.: Formulating a new express minibus service design problem as a clustering problem, Transportation Science 49 85-98, 2014.

18. Psaraftis, H.N.: Dynamic vehicle routing: Status and prospects, Annals of Operations Research 61 143-164, 1995.

19. Ritzinger, U., Puchinger, J., Hartl, R.F.: A survey on dynamic and stochastic vehicle routing problems, International Journal of Production Research 54 215-231, 2016.

20. Verdonck, L., Caris, A., Ramaekers, K., Janssens, G.K.: Collaborative logistics from the perspective of road transportation companies, Transport Reviews 33 700-719, 2013.

21. Waller, M.A., Fawcett, S.E.: Data science, predictive analytics, and big data: a revolution that will transform supply chain design and management, Journal of Business Logistics 34 77-84, 2013. 